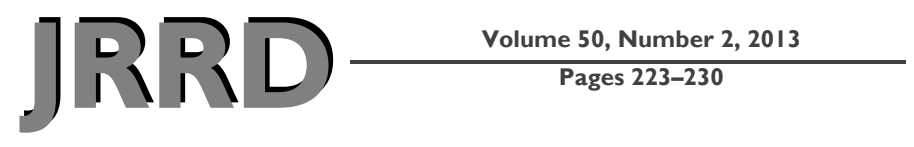

\title{
Laser light visual cueing for freezing of gait in Parkinson disease: A pilot study with male participants
}

\author{
Lisette Bunting-Perry, PhD, RN; ${ }^{1}$ Meredith Spindler, MD; ${ }^{1-2}$ Keith M. Robinson, MD; ${ }^{1,3}$ Joseph Noorigian, MPH; $^{1}$ \\ Heather J. Cianci, PT, MS, GCS; ${ }^{4}$ John E. Duda, MD ${ }^{1-2^{*}}$ \\ ${ }^{1}$ Parkinson's Disease Research, Education, and Clinical Center, Philadelphia Department of Veterans Affairs Medical \\ Center, Philadelphia, PA; Departments of ${ }^{2}$ Neurology and ${ }^{3}$ Physical Medicine and Rehabilitation, Perelman School of \\ Medicine at the University of Pennsylvania, Philadelphia, PA; ${ }^{4}$ Dan Aaron Parkinson's Rehabilitation Center, Pennsyl- \\ vania Hospital, Good Shepherd Penn Partners, Philadelphia, PA
}

\begin{abstract}
Freezing of gait (FOG) is a debilitating feature of Parkinson disease (PD). In this pilot study, we sought to assess the efficacy of a rolling walker with a laser beam visual cue to treat FOG in PD patients. We recruited 22 subjects with idiopathic PD who experienced on- and off-medication FOG. Subjects performed three walking tasks both with and without the laser beam while on medications. Outcome measures included time to complete tasks, number of steps, and number of FOG episodes. A crossover design allowed within-group comparisons between the two conditions. No significant differences were observed between the two walking conditions across the three tasks. The laser beam, when applied as a visual cue on a rolling walker, did not diminish FOG in this study.
\end{abstract}

Clinical Trial Registration: ClinicalTrials.gov; NCT01502995, "Laser Light Visual Cueing For Freezing Of Gait In Parkinson's Disease”; http://www.clinicaltrials.gov

Key words: assistive devices, freezing of gait, gait disorders, gait hesitation, gait ignition failure, laser light, Parkinson disease, rolling walker, visual cue, walker, U-Step.

\section{INTRODUCTION}

Freezing of gait (FOG) is a disabling phenomenon that affects anywhere from 30 to 60 percent of individuals with
Parkinson disease (PD) in cross-sectional studies [1-3]. It leads to impaired mobility, falls, and compromised activities of daily living and quality of life [4-6]. Although FOG improves with dopaminergic therapy in many patients, as motor fluctuations emerge, FOG becomes increasingly frequent and the response to therapy can wane [7]. Furthermore, many patients never experience relief of their FOG with dopaminergic therapy despite response of other motor symptoms, indicating that the pathophysiology underlying this symptom is not limited to the dopaminergic motor loop. Additionally, FOG is often provoked by certain environments or situations, such as initiating gait, navigating doorways and other narrow spaces, turning, and approaching a destination, implying the additional involvement of

Abbreviations: FOG $=$ freezing of gait; FOGQ $=$ Freezing of Gait Questionnaire; MMSE = Mini Mental Status Examination; PADRECC = Parkinson's Disease Research, Education, and Clinical Center; PD = Parkinson disease; UPDRS = Unified Parkinson's Disease Rating Scale, VA = Department of Veterans Affairs.

*Address all correspondence to John E. Duda, MD; Director, Parkinson's Disease Research, Education, and Clinical Center, Philadelphia Department of Veterans Affairs Medical Center, 3900 Woodland Ave, Philadelphia, PA 19104; 215823-5934; fax: 215-823-5815. Email: John.duda@va.gov http://dx.doi.org/10.1682/JRRD.2011.12.0255 
sensory systems. Despite FOG's prominence among the disabling symptoms of advancing PD, no interventions have been clearly proven to reduce on-medication FOG.

Further supporting the role of diverse and nondopaminergic circuitry in the mechanism of FOG, visual and auditory cueing strategies have been reported to ameliorate FOG and other gait deficits in patients with PD [8-12]. High-contrast, transverse line visual cues on the floor have been found to improve stride length and velocity, among other measures, in patients with PD in several trials, one of which also demonstrated reduced FOG episodes [11,13-18]. Another of these trials demonstrated similar benefits with a laser beam visual cue [15]. A recent trial conducted by Donovan et al. used both a laser light cane and walker in a crossover study [19]. A modest but significant improvement in the primary outcome, the Freezing of Gait Questionnaire (FOGQ), was observed with use of the laser light cue.

Similar to this recent study, we studied the U-Step rolling walker (In-Step Mobility Products, Inc; Skokie, Illinois), which also uses a laser light beam activated by the patient as a visual cue to break FOG in patients who have FOG in both the on- and off-medication states. To assess for FOG episodes objectively, we used an obstacle course incorporating the previously listed environmental and situational features known to provoke FOG to measure primary outcomes and recorded both time to completion and number of FOG episodes. Given the above data regarding visual cues, we hypothesized that use of the laser light beam with appropriate instruction would reduce FOG during three walking tasks in comparison with a rolling walker alone.

\section{METHODS}

\section{Participants}

A convenience sample of 22 subjects was recruited consecutively over 1 year from the Parkinson's Disease Research, Education, and Clinical Center (PADRECC) at the Philadelphia Department of Veterans Affairs (VA) Medical Center. Subjects who experienced FOG were referred to study personnel by their PADRECC clinician. Inclusion criteria were the following: idiopathic PD confirmed by a movement disorders neurologist; a MiniMental Status Examination (MMSE) [20] score $\geq 24$ to ensure competence to participate in informed consent and the ability to learn procedurally the use of the rolling walker with laser beam; functional visual acuity for recognizing the laser beam on the rolling walker; FOG in both on- and off-medication states, as indicated by a score of $\geq 2$ on Item 14 of the Unified Parkinson's Disease Rating Scale (UPDRS) and/or $\geq 1$ on Item 3 of the FOGQ [2122]; and ability to ambulate for at least household distances with or without an assistive device, as indicated by scoring 0,1 , or 2 on Items 15 and 29 of the UPDRS.

\section{Equipment}

A four-wheeled rolling walker with a horizontal red laser beam at ankle level (U-Step LaserLite) was used. The laser beam was triggered by an on/off button on the right handle of the rolling walker. The walker was equipped with reverse brakes, requiring the subject to squeeze the hand brake to walk and release the hand brake to stop.

\section{Procedures}

Patients were screened for FOG and study eligibility via medical record review or history and physical examination at initial visits. They were advised to take their dopaminergic medications 1 hour before their study appointment, allowing the testing to occur in the on-medication state to optimize motor control and reduce fatigue. However, if the study subjects progressed to the off-medication state during testing, they were instructed to take their next dose of medications and testing was resumed when the on-medication state was reached. Thus, all patients were tested in the onmedication state, controlling for any dopaminergic therapy effects on FOG. Participants were rated by investigators using the UPDRS, Hoehn \& Yahr Scale [23], Schwab \& England Activities of Daily living Scale, MMSE, and FOGQ, as summarized in Table 1.

Participants were trained in the use of the rolling walker with the laser beam before testing to minimize practice effects. When episodes of FOG occurred, study subjects were instructed to press the on/off button to initiate the laser beam, look down, and step over the laser beam to re-initiate gait while concurrently squeezing the hand brake release. Once the FOG episode was aborted, subjects were instructed to turn off the laser beam. Training involved practicing with the rolling walker after instruction on its use until the subject was comfortable using the walker and then completing the three walking tasks (described subsequently) at a relaxed pace and without measurement of outcome measures. After completion of training, subjects rested for a few minutes before 
beginning the walking tasks with outcome measure to minimize the effects of fatigue. Patients were then asked to complete three walking tasks both without the light beam (Trial A) and with the light beam (Trial B). Subjects were randomized to perform either Trial A or Trial B first in order to control for fatigue and practice effects. No coaching was provided by study staff during the walking tasks. The trials took place in the PADRECC using a 7.75 ft-wide, $70 \mathrm{ft}$-long hallway and six examination rooms, within which patients had $12 \mathrm{ft}$ of walking distance to be covered in a cul-de-sac fashion. The three walking tasks contained environmental stimuli known to provoke FOG. The most simple task involved walking down a wide hallway and turning to sit in the chair ("Straight”; Table 2); the second, more complex task involved initiation in the hallway, consecutively entering and exiting six examination rooms, and ending by sitting in a chair in the hallway ("Rooms"; Table 2); and the third task involved walking down a hallway with cones, having to stop and restart at each cone ("Cones"; Table 2).

All subjects were videotaped to validate the FOG episodes and the blinded rater's observed measures. Three outcome measures were observed and recorded during each task: total time to complete, number of FOG episodes, and number of steps taken during each walking task. We hypothesized that the mean completion time, mean number of FOG episodes, and mean number of steps would be reduced in the trials with the laser beam.

Table 1.

Characteristics of study participants. Data presented as mean \pm standard deviation.

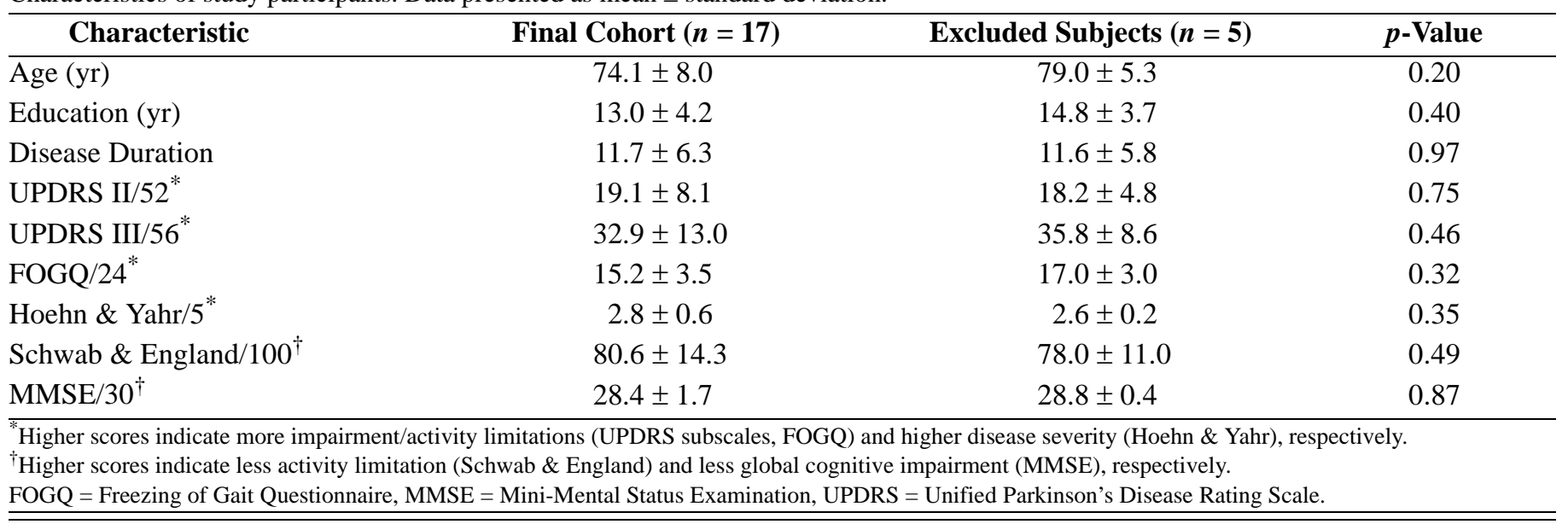

Table 2.

Outcome measures $(n=17)$. Data presented as mean \pm standard deviation.

\begin{tabular}{lccc}
\hline \multicolumn{1}{c}{ Task } & Trial A: Without Light & Trial B: With Light & p-Value \\
\hline Rooms & $178.4 \pm 151.5$ & $184.0 \pm 157.5$ & 0.74 \\
Time to Complete (s) & $171.8 \pm 74.6$ & $180.6 \pm 73.8$ & $7.8 \pm 7.8$ \\
Steps to Complete (No.) & $6.2 \pm 4.8$ & & \\
FOG Episodes (No.) & & $49.8 \pm 40.8$ \\
Straight & $42.0 \pm 27.6$ & $54.3 \pm 18.5$ \\
Time to Complete (s) & $55.5 \pm 21.8$ & $1.2 \pm 2.3$ \\
Steps to Complete (No.) & $0.94 \pm 1.9$ & & 0.08 \\
FOG Episodes (No.) & & $76.6 \pm 60.0$ \\
Cones & $71.5 \pm 51.6$ & $67.1 \pm 20.2$ \\
Time to Complete (s) & $78.8 \pm 53.6$ & $1.8 \pm 3.3$ \\
Steps to Complete (No.) & $2.1 \pm 3.6$ & 0.84 \\
FOG Episodes (No.) & & & 0.55 \\
FOG = freezing of gait. & & 0.67 \\
\hline \hline
\end{tabular}




\section{Statistical Analysis}

Baseline characteristics of subgroups were compared using Mann-Whitney rank-sum tests to determine statistically significant differences. Differences between the trials with and without laser light were assessed using the Wilcoxon signed-rank test, given the small sample size and non-normal distribution of data. A significance threshold of 0.05 was used.

\section{RESULTS}

Of 109 subjects screened, 22 met the inclusion criteria and were willing to participate. Excluded participants were those who were too frail to complete the walking tasks $(n=19)$; those who were too cognitively impaired to learn the procedures $(n=24)$; and those who were eligible but did not want to participate $(n=44)$, most of whom were deterred by the inconvenience of travel. All patients were male, and their baseline characteristics are summarized in Table 1. Only 3 of the 22 participants had been issued a rolling walker before their participation in this study. During the study, 17 of the 22 participants experienced on-medication FOG, while 5 did not. These 5 subjects were excluded from the final analysis, because the effect of the intervention depended on the occurrence of FOG. Descriptive characteristics of the entire cohort, the final cohort of 17 subjects, and the excluded subjects were similar and are summarized in Table $\mathbf{1}$. The results, as summarized in Table 2, are based on the performances of the 17 subjects who did demonstrate FOG. No significant differences in the three performance measures were observed between the two study conditions, with and without laser light.

\section{DISCUSSION}

This study did not demonstrate a beneficial effect of the red laser beam visual cue on FOG. Despite efforts to objectively assess outcomes and control for confounders such as medication regimens, fatigue, and learning using a crossover design, this study demonstrates many limitations and pitfalls that exist when studying FOG, and these may prove instructive for future investigators.

Our choice to use objective gait measures as outcomes, rather than a subjective measure such as the FOGQ, ensured uniform conditions and freedom from recall bias. However, despite endorsing FOG, only 17 of our 22 subjects demonstrated FOG during the walking tasks, making it impossible to detect any effects of the laser light beam in nearly a quarter of our cohort. Furthermore, the variability in our subjects' performance during the tasks, whether with the laser beam or without, was greater than we anticipated, further reducing our study's power. This may have been partially due to motor fluctuations that occurred despite our efforts to ensure that patients were in the on-medication state during testing. This variability, along with the small sample size and the lack of FOG in a quarter of our cohort, may have contributed to the null results. Similar difficulties in studying FOG in the on-medication state have been encountered by other investigators, mainly because of the unpredictability of FOG despite provocative environmental conditions [24]. Nieuwboer et al. studied various cueing strategies on turn speed in 60 reported freezers, but only 8 subjects actually froze during the complex turning task [25]. Although testing patients in the off-medication state would have increased the rate of FOG in our study, this was precluded by the severity of gait impairment while off medications and would have been less clinically relevant. Of note, a recently published study by Donovan et al. used the FOGQ as the primary outcome measure and demonstrated a modest but significant reduction of FOG in 26 patients using the U-Step cane or walker equipped with a laser light beam [19]. An objective secondary outcome measure-a timed gait test involving walking $20 \mathrm{ft}$, going through a doorway, turning, and walking back another $20 \mathrm{ft}$ - did not improve. Whether this discrepancy is due to insensitivity of objective measures or recall bias of subjective measures is unclear.

Despite these limitations of our methods, our negative findings are consistent with a few other clinical trials of visual cueing strategies for FOG. Kompoliti et al. found that on-medication FOG in PD subjects was inconsistently responsive to an inverted walking stick with a perpendicular ankle-level outrigger and a laser beam that projected onto the floor [26]. Cubo et al. found that in PD patients in the on-medication state, both standard and wheeled walkers significantly slowed walking speed, standard walkers induced significantly more FOG, and rolling walkers with a laser beam did not influence FOG [27]. In their investigation using an inverted walking stick to interrupt FOG, Dietz et al. reported that only two of the eight subjects had an improved walking time and decreased number of FOG episodes, while the study 
group as a whole had a significantly increased walking time and number of FOG episodes [9]. Thus, the authors proposed that a certain subgroup of PD individuals may benefit from visual cueing.

In contrast to the studies above, there are several reports of successful reduction of FOG with visual cues. Improved gait in PD in response to visual cues was demonstrated as early as 1967 and has been replicated since [14]. Azulay et al. demonstrated that parallel lines on the walking surface increased stride length and gait velocity in 16 PD patients [14]. Similarly, in a trial of 14 patients with PD and 14 controls conducted by Lewis et al., two different visual cues were both associated with improvements of stride length and velocity in PD patients compared with control subjects: transverse white lines on the floor and two red laser beam lines continually projected onto the floor one stride length apart [15]. Jiang and Norman reported 14 patients with PD, 7 with FOG, who demonstrated increased first and second stride lengths, greater push-off force, and higher velocity with highcontrast transverse line visual cues on the floor [11]. Applying these observations to FOG specifically, Lee et al. recently published a trial in which white stripes on the floor significantly increased velocity and stride length and decreased cadence and freezing episodes in PD patients with FOG [16]. Dunne et al. reported on three PD subjects who were extensively trained in the use of a visual cue to break freezing and whose FOG was thereafter effectively aborted using an upturned walking stick [10]. Perhaps more thorough training with visual cues than was done in our study is needed to observe benefits.

An important feature of the interventions used in the above studies, as opposed to the current study, is that subjects were not required to activate the visual cue. The concurrent use of the assistive device and the activation of the laser beam represents a dual task that is more demanding of attentional resources than when walking unassisted or with an assistive device alone. Such dual tasks have consistently been demonstrated to worsen gait parameters in PD patients [28-30]. This phenomenon may have been particularly important in our study because most subjects were using a walker for the first time, and they may have been stopping in anticipation of FOG to activate the laser beam. The use of transverse lines on the floor, while less translatable to subjects' daily lives than the U-Step walker, avoids this phenomenon. Perhaps the continual projection of the beam, as used by Donovan et al. [19], or of two laser beams one stride length apart, as employed by Lewis et al. [15], would reduce dual tasking and thereby improve efficacy. Also intriguing are the results of a pilot study of seven patients by Bryant et al., who showed that compared with no light beam, use of a green light beam but not a red light beam improved FOG in patients off medication. [31]. Patients on medication did improve with the red light beam, but not as much as with the green light. Whereas the transverse lines used in most of the justdescribed studies were white, the laser beams, which have had less effect, have been red; more studies are needed to clarify the role of color in visual cueing.

Several limitations to studying interventions for FOG have been illustrated by this study and can inform future studies on this disabling symptom of PD. Such studies should consider minimizing dual tasking by using continuous visual stimuli; ensuring adequate training with visual cues and assistive devices; testing various colors of laser beams; and collecting data regarding other features of the subjects' disease, with the goal of characterizing any subgroup that may respond. In the meantime, because the evidence from other studies suggests that some patients do respond, the use of devices with visual cues should not be abandoned and should be assessed on an individual basis.

\section{CONCLUSIONS}

In this study, the use of a laser beam on a rolling walker did not reduce FOG in a cohort of male PD patients. Future studies should consider including duration of FOG episodes among objective outcome measures, minimizing dual tasking, ensuring adequate training with cues and devices, and identifying potential subgroups of patients who are more likely to benefit.

\section{ACKNOWLEDGMENTS}

\section{Author Contributions:}

Study concept or design: L. Bunting-Perry, K. M. Robinson, J. E. Duda. Acquisition of data: L. Bunting-Perry, K. M. Robinson. Analysis or interpretation of data: L. Bunting-Perry, M. Spindler, K. M. Robinson, J. Noorigian, H. J. Cianci, J. E. Duda.

Statistical analysis: M. Spindler, J. Noorigian. Drafting/revising manuscript: L. Bunting-Perry, M. Spindler, H. J. Cianci.

Critical revision of manuscript for important intellectual content: K. M. Robinson, J. E. Duda. 
Study supervision: K. M. Robinson, J. E. Duda.

Financial Disclosures: The authors have declared that no competing interests exist. In-Step Mobility Products was not involved in the study design; collection, analysis, and interpretation of data; writing of the report; or decision to submit the report for publication.

Funding/Support: This work was supported by a PADRECC Pilot Project Award from the Philadelphia VA Medical Center to Dr. BuntingPerry.

Additional Contributions: The rolling walker with laser beam used in this study was donated by In-Step Mobility Products. Dr. BuntingPerry is now also affiliated with Ipsen Biopharmaceuticals, Inc.

Institutional Review: The study was approved by the Philadelphia VA Medical Center Institutional Review Board, and informed consent was obtained from each subject.

Participant Follow-Up: The authors do not plan to inform participants of the publication of this study.

Disclaimers: The content of this report does not reflect the view of the VA or the U.S. Government.

\section{REFERENCES}

1. Giladi N, McMahon D, Przedborski S, Flaster E, Guillory S, Kostic V, Fahn S. Motor blocks in Parkinson's disease. Neurology. 1992;42(2):333-39. [PMID:1736161]

http://dx.doi.org/10.1212/WNL.42.2.333

2. Lamberti P, Armenise S, Castaldo V, de Mari M, Iliceto G, Tronci P, Serlenga L. Freezing gait in Parkinson's disease. Eur Neurol. 1997;38(4):297-301. [PMID:9434089] http://dx.doi.org/10.1159/000113398

3. Macht M, Kaussner Y, Möller JC, Stiasny-Kolster K, Eggert KM, Krüger HP, Ellgring H. Predictors of freezing in Parkinson's disease: a survey of 6,620 patients. Mov Disord. 2007;22(7):953-56. [PMID:17377927]

http://dx.doi.org/10.1002/mds.21458

4. Okuma Y. Freezing of gait in Parkinson's disease. J Neurol. 2006;253(Suppl 7):vii27-32. [PMID:17131225]

http://dx.doi.org/10.1007/s00415-006-7007-2

5. Bloem BR, Hausdorff JM, Visser JE, Giladi N. Falls and freezing of gait in Parkinson's disease: a review of two interconnected, episodic phenomena. Mov Disord. 2004; 19(8):871-84. [PMID:15300651] http://dx.doi.org/10.1002/mds.20115

6. Okuma Y, Yanagisawa N. The clinical spectrum of freezing of gait in Parkinson's disease. Mov Disord. 2008; 23(Suppl 2):S426-30. [PMID:18668623]

http://dx.doi.org/10.1002/mds.21934

7. Schaafsma JD, Balash Y, Gurevich T, Bartels AL, Hausdorff JM, Giladi N. Characterization of freezing of gait subtypes and the response of each to levodopa in Parkinson’s disease. Eur J Neurol. 2003;10(4):391-98.

[PMID:12823491]

http://dx.doi.org/10.1046/j.1468-1331.2003.00611.x
8. Rubinstein TC, Giladi N, Hausdorff JM. The power of cueing to circumvent dopamine deficits: a review of physical therapy treatment of gait disturbances in Parkinson's disease. Mov Disord. 2002;17(6):1148-60. [PMID:12465051] http://dx.doi.org/10.1002/mds.10259

9. Dietz MA, Goetz CG, Stebbins GT. Evaluation of a modified inverted walking stick as a treatment for parkinsonian freezing episodes. Mov Disord. 1990;5(3):243-47. [PMID:2388642] http://dx.doi.org/10.1002/mds.870050311

10. Dunne JW, Hankey GJ, Edis RH. Parkinsonism: upturned walking stick as an aid to locomotion. Arch Phys Med Rehabil. 1987;68(6):380-81. [PMID:3592954]

11. Jiang Y, Norman KE. Effects of visual and auditory cues on gait initiation in people with Parkinson's disease. Clin Rehabil. 2006;20(1):36-45. [PMID:16502748] http://dx.doi.org/10.1191/0269215506cr925oa

12. Morris ME, Iansek R, Galna B. Gait festination and freezing in Parkinson's disease: pathogenesis and rehabilitation. Mov Disord. 2008;23(Suppl 2):S451-60. [PMID:18668618] http://dx.doi.org/10.1002/mds.21974

13. Morris ME, Iansek R, Matyas TA, Summers JJ. The pathogenesis of gait hypokinesia in Parkinson's disease. Brain. 1994;117(Pt 5):1169-81. [PMID:7953597] http://dx.doi.org/10.1093/brain/117.5.1169

14. Azulay JP, Mesure S, Amblard B, Blin O, Sangla I, Pouget J. Visual control of locomotion in Parkinson's disease. Brain. 1999;122(Pt 1):111-20. [PMID:10050899] http://dx.doi.org/10.1093/brain/122.1.111

15. Lewis GN, Byblow WD, Walt SE. Stride length regulation in Parkinson's disease: the use of extrinsic, visual cues. Brain. 2000;123(Pt 10):2077-90. [PMID:11004125] http://dx.doi.org/10.1093/brain/123.10.2077

16. Lee SJ, Yoo JY, Ryu JS, Park HK, Chung SJ. The effects of visual and auditory cues on freezing of gait in patients with Parkinson disease. Am J Phys Med Rehabil. 2012;91(1):2-11. [PMID:22157432] http://dx.doi.org/10.1097/PHM.0b013e31823c7507

17. Morris ME, Iansek R, Matyas TA, Summers JJ. Ability to modulate walking cadence remains intact in Parkinson's disease. J Neurol Neurosurg Psychiatry. 1994;57(12): 1532-34. [PMID:7798986]

http://dx.doi.org/10.1136/jnnp.57.12.1532

18. Morris ME, Iansek R, Matyas TA, Summers JJ. Stride length regulation in Parkinson's disease. Normalization strategies and underlying mechanisms. Brain. 1996;119(Pt 2):551-68. [PMID:8800948]

http://dx.doi.org/10.1093/brain/119.2.551

19. Donovan S, Lim C, Diaz N, Browner N, Rose P, Sudarsky LR, Tarsy D, Fahn S, Simon DK. Laserlight cues for gait freezing in Parkinson's disease: an open-label study. Parkinsonism Relat Disord. 2011;17(4):240-45. 
BUNTING-PERRY et al. Laser light for freezing of gait

\section{[PMID:20817535]}

http://dx.doi.org/10.1016/j.parkreldis.2010.08.010

20. Folstein MF, Folstein SE, McHugh PR. "Mini-mental state". A practical method for grading the cognitive state of patients for the clinician. J Psychiatr Res. 1975;12(3):189-98. [PMID:1202204] http://dx.doi.org/10.1016/0022-3956(75)90026-6

21. Giladi N, Shabtai H, Simon ES, Biran S, Tal J, Korczyn $\mathrm{AD}$. Construction of freezing of gait questionnaire for patients with Parkinsonism. Parkinsonism Relat Disord. 2000;6(3):165-70. [PMID:10817956] http://dx.doi.org/10.1016/S1353-8020(99)00062-0

22. Giladi N, Tal J, Azulay T, Rascol O, Brooks DJ, Melamed E, Oertel W, Poewe WH, Stocchi F, Tolosa E. Validation of the freezing of gait questionnaire in patients with Parkinson's disease. Mov Disord. 2009;24(5):655-61.

[PMID:19127595]

http://dx.doi.org/10.1002/mds.21745

23. Hoehn MM, Yahr MD. Parkinsonism: onset, progression and mortality. Neurology. 1967;17(5):427-42.

[PMID:6067254] http://dx.doi.org/10.1212/WNL.17.5.427

24. Nieuwboer A, Giladi N. The challenge of evaluating freezing of gait in patients with Parkinson's disease. Br J Neurosurg. 2008;22(Suppl 1):S16-18. [PMID:19085348] http://dx.doi.org/10.1080/02688690802448376

25. Nieuwboer A, Baker K, Willems AM, Jones D, Spildooren J, Lim I, Kwakkel G, Van Wegen E, Rochester L. The short-term effects of different cueing modalities on turn speed in people with Parkinson's disease. Neurorehabil Neural Repair. 2009;23(8):831-36. [PMID:19491396] http://dx.doi.org/10.1177/1545968309337136

26. Kompoliti K, Goetz CG, Leurgans S, Morrissey M, Siegel IM. “On” freezing in Parkinson's disease: resistance to visual cue walking devices. Mov Disord. 2000;15(2):309-12. [PMID:10752582] http://dx.doi.org/10.1002/1531-8257(200003)15:2<309::AIDMDS1016>3.0.CO;2-P

27. Cubo E, Moore CG, Leurgans S, Goetz CG. Wheeled and standard walkers in Parkinson's disease patients with gait freezing. Parkinsonism Relat Disord. 2003;10(1):9-14.

[PMID:14499200]

http://dx.doi.org/10.1016/S1353-8020(03)00060-9

28. O’Shea S, Morris ME, Iansek R. Dual task interference during gait in people with Parkinson disease: effects of motor versus cognitive secondary tasks. Phys Ther. 2002; 82(9):888-97. [PMID:12201803]

29. Rochester L, Hetherington V, Jones D, Nieuwboer A, Willems AM, Kwakkel G, Van Wegen E. Attending to the task: interference effects of functional tasks on walking in Parkinson's disease and the roles of cognition, depression, fatigue, and balance. Arch Phys Med Rehabil. 2004;85(10): 1578-85. [PMID:15468014] http://dx.doi.org/10.1016/j.apmr.2004.01.025

30. Yogev G, Giladi N, Peretz C, Springer S, Simon ES, Hausdorff JM. Dual tasking, gait rhythmicity, and Parkinson's disease: which aspects of gait are attention demanding? Eur J Neurosci. 2005;22(5):1248-56. [PMID:16176368] http://dx.doi.org/10.1111/j.1460-9568.2005.04298.x

31. Bryant MS, Rintala DH, Lai EC, Protas EJ. A pilot study: influence of visual cue color on freezing of gait in persons with Parkinson's disease. Disabil Rehabil Assist Technol. 2010;5(6):456-61. [PMID:20545563] http://dx.doi.org/10.3109/17483107.2010.495815

Submitted for publication December 30, 2011. Accepted in revised form June 22, 2012.

This article and any supplementary material should be cited as follows:

Bunting-Perry L. Spindler M, Robinson KM, Noorigian J, Cianci HJ, Duda JE. Laser light visual cueing for freezing of gait in Parkinson disease: A pilot study with male participants. J Rehabil Res Dev. 2013;50(2):223-30. http://dx.doi.org/10.1682/JRRD.2011.12.0255

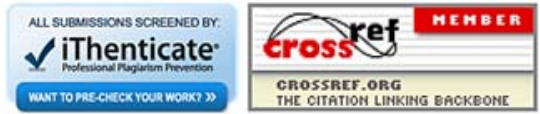


